\title{
Insulin-like growth factor 1 inhibits autophagy of human colorectal carcinoma drug-resistant cells via the protein kinase B/mammalian target of rapamycin signaling pathway
}

\author{
SHUOMIN WANG ${ }^{1,2}$ and KANGSHENG GU ${ }^{1}$ \\ ${ }^{1}$ Department of Oncology, The First Affiliated Hospital of Anhui Medical University. Hefei, Anhui 230022; \\ ${ }^{2}$ Department of Oncology, The Fourth Affiliated Hospital of Anhui Medical University, Hefei, Anhui 230032, P.R. China
}

Received April 25, 2016; Accepted March 30, 2017

DOI: $10.3892 / \mathrm{mmr} .2017 .8272$

\begin{abstract}
Insulin-like growth factor 1 (IGF-1) is reported to inhibit autophagy of human colorectal carcinoma cells (HCT); however, little is known regarding the mechanisms underlying the inhibitory effect of IGF-1 on autophagy in HCT resistant strains. The present study aimed to analyze the inhibitory effect of IGF-1 on the autophagy of HCT resistant strains and its potential underlying mechanisms. The viability and apoptosis of HCT-8 colon cancer cells were analyzed, and expression levels of relevant genes and proteins were investigated using reverse transcription-quantitative polymerase chain reaction and western blot analysis, respectively. Treatment of cells with IGF-1 induced apoptosis. IGF-1 treatment activated protein kinase B (AKT), which may inhibit autophagy via the AKT/mammalian target of rapamycin signaling pathway. Following inhibition of autophagy, drug resistant cells became sensitive to apoptosis induced by 5 -fluorouracil.
\end{abstract}

\section{Introduction}

Colorectal cancer is one of the most common types of cancer worldwide and the incidence is increasing (1). Although progress has been made with regard to treatment of colorectal cancer, patient survival remains poor (2). Currently, the primary drugs used for the treatment of colorectal cancer are oxaliplatin and 5-fluorouracil (5-FU) (3). How autophagy determines and regulates the sensitivity of colorectal cancer cells to 5-FUremainsunclear and requires investigation.

Autophagy is a degradation process that has important roles in cellular homeostasis (4). Despite its simplicity,

Correspondence to: Dr Kangsheng Gu, Department of Oncology, The First Affiliated Hospital of Anhui Medical University, 218 Jixi Road, Hefei, Anhui 230022, P.R. China

E-mail: kangshenggu11@sina.com

Key words: insulin-like growth factor 1, human colorectal carcinoma cells, autophagy, protein kinase B, mammalian target of rapamycin evidence has demonstrated that autophagy is a highly complex process, involving protein degradation, organelle turnover and breakdown of cytoplasmic components during nutrient starvation or stress (5). Autophagy contributes to apoptosis when it is executed excessively or inefficiently (6). It has been suggested that autophagy serves a fundamental role in tumor progression (7-10).

Insulin-like growth factor-1 (IGF-1) has been reported to regulate cell survival, proliferation, differentiation and metabolism (11-13). IGF-1 has an inhibitory role on autophagy in various cell types, including human osteocarcinoma cells (11). However, little is known regarding the mechanisms underlying its inhibitory effect on autophagy in drug-resistant human colorectal carcinoma cells (HCT).

Therefore, the aim of the present study was to analyze the inhibitory effect of IGF-1 on autophagy in drug-resistant HCT, and its underlying mechanism.

\section{Materials and methods}

Cell culture. HCT-8 human HCT and HCT-8R5-FU-resistant HCT cells were obtained from Bogoo Biomart (Shanghai, China) The cells were cultured in RPMI-1640 medium (Gibco; Thermo Fisher Scientific, Inc., Waltham, MA, USA) containing 10\% fetal bovine serum (Gibco; Thermo Fisher Scientific, Inc.), $2 \mathrm{mM}$ L-glutamine, $100 \mathrm{U} / \mathrm{ml}$ penicillin and $100 \mathrm{ng} / \mathrm{ml}$ streptomycin at $37^{\circ} \mathrm{C}$ in a humidified atmosphere of $95 \%$ air. The medium was replaced every 2 days.

Autophagy analysis using the DsRed-microtubule-associated protein 1A/1B-light chain 3 (LC3)reporter. To develop an autophagy reporter, DsRed protein was fused with the $\mathrm{N}$-terminus of the human LC3 protein through transfecting $293 \mathrm{~T}$ cells with a lentivirus. Recombinant lentiviruses expressing the DsRed-LC3 reporter were generated and applied to infect target cells (14).

Apoptosis assays. For apoptosis assays, cells were seeded at $5 \times 10^{5}$ cells $/ \mathrm{ml}$ in triplicate and starved with serum-free medium for $24 \mathrm{~h}$, and subsequently treated with 10 or $50 \mathrm{nM}$ IGF-1 [Phoenix Biotech (Beijing) Co., Ltd., Beijing, China], 10 nM MK-2206 (Selleck Chemicals, Houston, TX, USA) or 
Table I. Primers used for quantitative polymerase chain reaction.

\begin{tabular}{lll}
\hline & \multicolumn{2}{c}{ Sequence (5'-3') } \\
\cline { 2 - 3 } Gene & \multicolumn{1}{c}{ Forward } & \multicolumn{1}{c}{ Reverse } \\
\hline ULK1 & CTGGTCCTCTTGCTTCCGTC & ACACCAGCCCAACAATTCC \\
BECN1 & TCCGGGCTCCCGAGG & TTCTCCTGGGTCTCTCCTG \\
Vps34 & GCTTAAGATCTGGAATGAATGGCT & TGCCAGGAGTTTTTGTGGT \\
Atg5 & GGGTCCCTCTTGGGGTACAT & ACCACACATCTCGAAGCACA \\
Atg7 & TGGTTACAAGCTTGGCTGCT & TCAAGAACCTGGTGAGGCAC \\
LC3B & AAGGCTTTCAGAGAGACCCTG & CCGTTTACCCTGCGTTTGTG \\
Atg16I & CCTGCAATAACAAATTGCTGGA & GCCTGTTGGTACGTCATGC \\
Atg4B & CTCATCTACCTGGACCCCCA & AGAATCTAGGGACAGGTTCAGGA \\
Atg12 & CTGTGTAATTGCGTCCCCT & GAAGCTGCAACACAGACTGC \\
GAPDH & AATGGGCAGCCGTTAGGAAA & GCGCCCAATACGACCAAATC \\
\hline
\end{tabular}

ULK1, unc-51 like autophagy activating kinase 1; BECN1, beclin-1; Vps34, phosphatidylinositol 3-kinase catalytic subunit type 3; Atg, autophagy-related; LC3B, microtubule-associated protein 1A/1B-light chain 3.

100 nM 3-methyladenine (MA; Sigma-Aldrich; Merck KGaA, Darmstadt, Germany) in combination with $10 \mu \mathrm{g} / \mathrm{ml} 5-\mathrm{FU}$ (Kyowa Hakko Kirin Co., Ltd., Tokyo, Japan). After 24 h, cells were stained with $5 \mu \mathrm{l}$ annexin V-fluorescein isothiocyanate and propidium iodide (PI; Invitrogen, Thermo Fisher Scientific, Inc.) for $15 \mathrm{~min}$ at room temperature and analyzed by flow cytometry (BD FACSCalibur ${ }^{\mathrm{TM}}$; BD Biosciences, Franklin Lakes, NJ, USA) using Flowjo software (version 10; Tree Star, Inc., Ashland, OR, USA).

Reverse transcription-quantitative polymerase chain reaction $(R T-q P C R)$ analysis. HCT-8R cells were treated with IGF-1, AKT inhibitor and 3-MA. Total RNA was extracted using the RNeasy kit (Qiagen GmbH, Hilden, Germany) and transcribed into cDNA with an RNA Reverse Transcriptase kit (Takara Bio, Inc., Otsu, Japan). qPCR was performed with a SYBR ${ }^{\circledR}$ Green PCR assay (Takara Bio, Inc.) according to the manufacturer's protocol $\left(95^{\circ} \mathrm{C}\right.$ for $1 \mathrm{~min}$, and 40 cycles of $95^{\circ} \mathrm{C}$ for $5 \mathrm{sec}$ and $60^{\circ} \mathrm{C}$ for $35 \mathrm{sec}$, followed by a final standard dissociation protocol), using the primers listed in Table I. Expression of GAPDH served as an internal control. The results were analyzed using the comparative $\mathrm{Cq}$ method $\left(2-{ }^{\Delta \Delta \mathrm{Cq}}\right)(15)$.

Western blot analysis. HCT-8R cells were treated with IGF-1, AKT inhibitor and 3-MA. Cells $\left(5 \times 10^{5}\right.$ cells $\left./ \mathrm{ml}\right)$ were lysed in radioimmunoprecipitation assay buffer (Beyotime Institute of Biotechnology, Haimen, China). The cell extracts were collected and diluted in SDS-loading buffer and denatured for $5 \mathrm{~min}$ at $95^{\circ} \mathrm{C}$, and protein determination was performed using a bicinchoninic acid assay. The samples (30 $\mu \mathrm{g})$ were separated using SDS-PAGE on a $12.5 \%$ gel and blotted onto $0.2 \mu \mathrm{m}$ polyvinylidene difluoride membranes (Bio-Rad Laboratories, Inc., Hercules, USA). Following blocking with 5\% skimmed milk powder in TBS containing Tween 20, membranes were incubated at $4^{\circ} \mathrm{C}$ overnightwithrabbit antibodies against LC3B (1:400, catalog no. ab48394; Abcam, Cambridge, UK), protein kinase B (1:400, AKT; catalog no. ab179463s; Abcam) and phosphorylated (p)-AKT (1:400, catalog no. ab81283; Abcam). Subsequently, membranes were incubated with a horseradish peroxidase-conjugated anti-rabbit secondary antibody at room temperature for $45 \mathrm{~min}$ (1:2,000; catalog no. sc-2357; Santa Cruz Biotechnology, Inc., Dallas, TX, USA) and proteins were visualized with an Enhanced Chemiluminescence system (PerkinElmer, Inc., Waltham, MA, USA). $\beta$-actin antibody (1:1,000; catalog no. sc-130656; Santa Cruz Biotechnology, Inc.) was used as an internal control.

Statistical analysis. Data are expressed as the mean \pm standard deviation. A one-way analysis of variance and Scheffé post hoc test was applied to investigate significant differences among multiple groups. Statistical analysis was performed using SPSS software version 11.0 (SPSS, Inc., Chicago, IL, USA). $\mathrm{P}<0.05$ was considered to indicate a statistically significant difference.

\section{Results}

Autophagy assay using the DsRed-LC3 reporter. Following culture for $24 \mathrm{~h}$, autophagy in the drug-resistant cells increased. Autophagic bodies decreased following IGF-1 treatment (Fig. 1); however, this was reversed upon the addition of an AKT inhibitor.

Apoptosis assays. Apoptosis was detected by annexin V-PI staining. In non-resistant cells, apoptosis was increased by 5-FU treatment, whereas the resistant strain exhibited reduced apoptosis at $24 \mathrm{~h}$ following 5-FU treatment (Fig. 2). In order to reduce the inhibitory effect of serum on autophagy, the cells were serum-starved for $24 \mathrm{~h}$, and subsequently cultured with 10 or $50 \mathrm{nM} \mathrm{IGF-1} \mathrm{and} \mathrm{5-FU} \mathrm{for} \mathrm{a} \mathrm{further} 24 \mathrm{~h}$. Eventually, apoptosis induced by 5-FU treatment was elevated by co-culture with IGF-1 in drug-resistant HCT-8R cells (Fig. 3). With the addition of the AKT inhibitor MK-2206 (10 nM) or autophagy agonist 3-MA (100 nM), apoptosis was decreased (Fig. 4). 

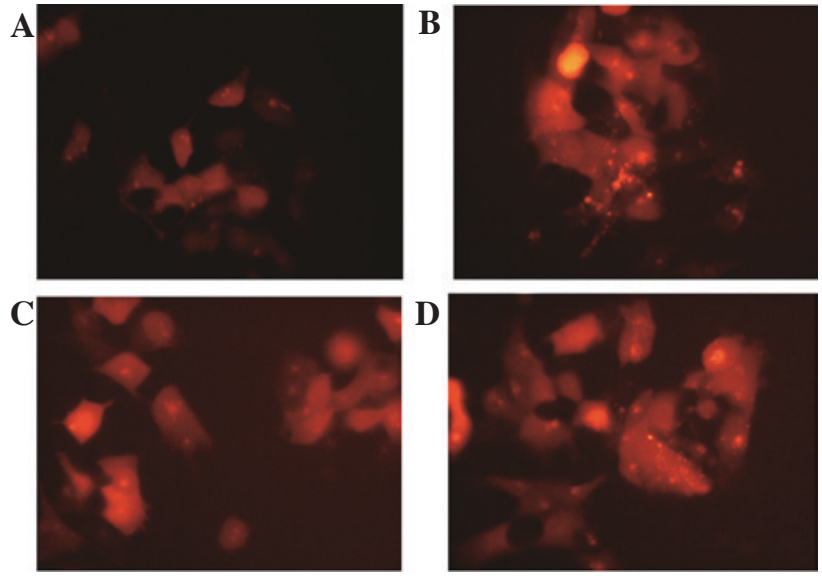

Figure 1. Autophagy assay. Autophagic bodies in (A) HCT-8 cells, (B) HCT-8R cells, (C) HCT-8R cells treated with IGF-1 and (D) HCT-8R cells treated with IGF-1 and an AKT inhibitor (magnification, x400). IGF-1, insulin-like growth factor 1; HCT, human colorectal carcinoma cells; R, resistant; AKT, protein kinase B.
A

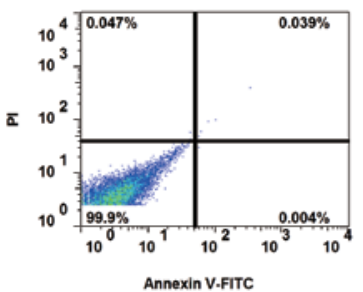

C

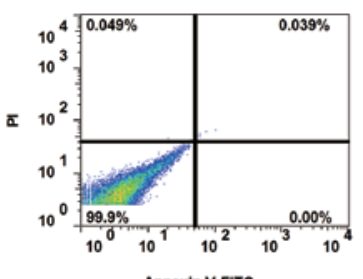

B

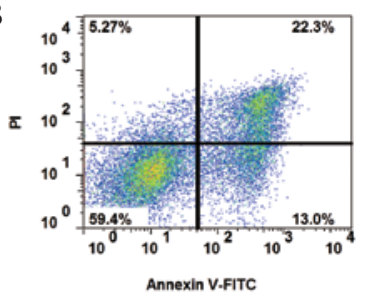

D

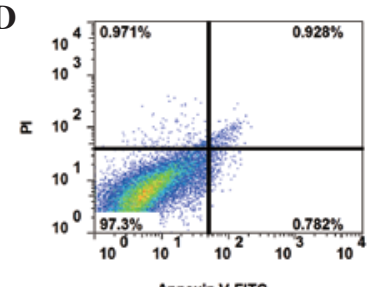

Figure 2.Apoptosis of non-resistant and drug-resistant cells following treatment with 5-FU. Annexin V-FITC and PI staining of (A) HCT-8 cells, (B) HCT-8 cells treated with 5-FU, (C) HCT-8R cells, and (D) HCT-8R cells treated with 5-FU. The upper and lower right quadrants indicate apoptotic cells. FITC, fluorescein isothiocyanate; PI, propidium iodide; 5-FU, 5-fluorouracil; HCT, human colorectal carcinoma cells; R, resistant.

RT-qPCR analysis. RT-qPCR was used to assess the effects of IGF-1 on the mRNA expression levels of autophagy-associated genes (Fig.5). In the initiation stage of autophagy, IGF-1 treatment downregulated the mRNA expression levels of the autophagy-associated genes unc-51 like autophagy activating kinase 1 (ULK1), beclin-1(Becn1) and phosphatidylinositol 3-kinase catalytic subunit type 3 (Vps34) in HCT-8cells (Fig. 5A). Treatment with IGF-1 and the AKT inhibitor MK-2206 significantly increased the mRNA expression levels of these genes; however, treatment with IGF-1 and 3-MA had no significant effect. In the elongation stage, a similar pattern was observed in the mRNA expression levels of autophagy related (Atg)5, Atg7 and Lc3b (Fig. 5B). In the expansion stage, IGF-1 downregulated the mRNA expression levels of Atg4b, Atg161 and Atg12, whereas addition of MK-2206 eliminated the inhibitory effect of IGF-1 (Fig. 5C).
A

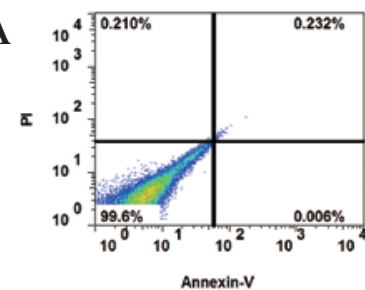

B

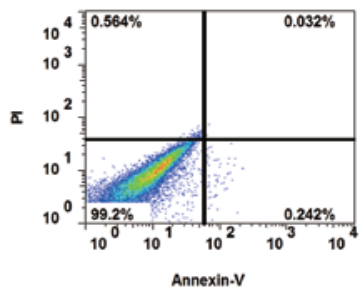

C

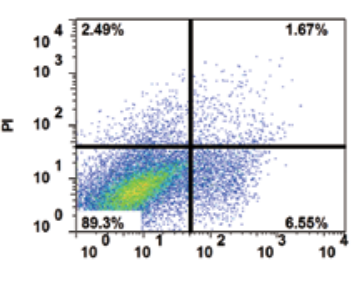

D

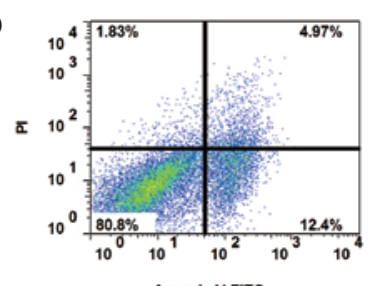

Figure 3. Apoptosis of drug-resistant cells following IGF-1 treatment Annexin V-FITC and PI staining of (A) HCT-8R cells treated with $10 \mathrm{nM}$ IGF-1, (B) HCT-8R cells treated with 50 nMIGF-1, (C) HCT-8R cells treated with $10 \mathrm{nM}$ IGF-1 and 5-FU, and (D) HCT-8R cells treated with $50 \mathrm{nM}$ IGF-1 and 5-FU.The upper and lower right quadrants indicate apoptotic cells. IGF-1, insulin-like growth factor 1; FITC, fluorescein isothiocyanate; PI, propidium iodide; HCT, human colorectal carcinoma cells; R, resistant; 5-FU, 5-fluorouracil.
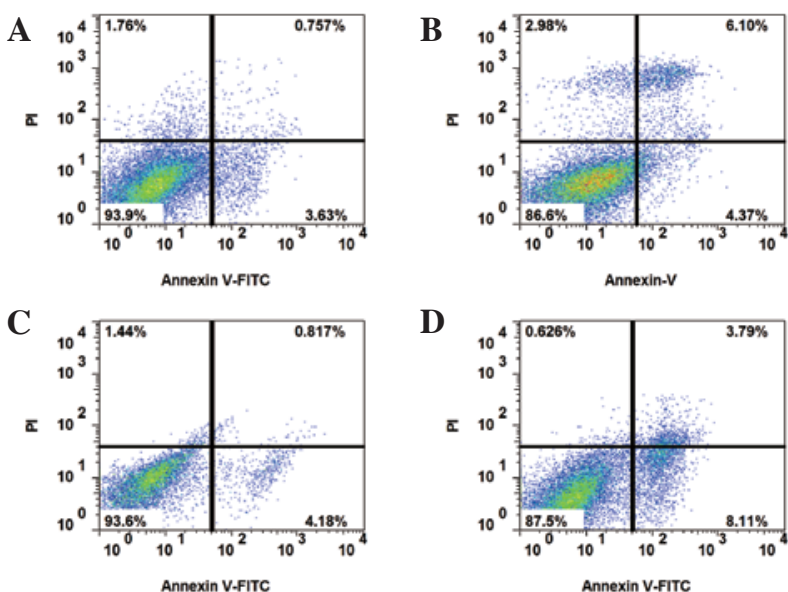

Figure 4. Apoptosis of drug-resistant cells following IGF-1 and AKT inhibitor treatment. Annexin V-FITC and PI staining of (A) HCT-8R cells treated with $10 \mathrm{nM}$ IGF-1, 5-FU and the AKT inhibitor MK-2206, (B) HCT-8R cells treated with $10 \mathrm{nM}$ IGF-1, 5-FU and the autophagy agonist3-MA, (C) HCT-8R cells treated with $50 \mathrm{nM}$ IGF-1, 5-FU and MK-2206, and (D) HCT-8R cells treated with $50 \mathrm{nM}$ IGF-1, 5-FU and 3-MA. The upper and lower right quadrants indicate apoptotic cells.IGF-1, insulin-like growth factor 1; AKT, protein kinase B; FITC, fluorescein isothiocyanate; PI, propidium iodide; HCT, human colorectal carcinoma cells; R, resistant; 5-FU, 5-fluorouracil; 3-MA, 3-methyladenine.

Western blot analysis. As presented in Fig. 6A, LC3 protein expression levels increased following treatment with IGF-1, and decreased with the addition of IGF-1 and the AKT inhibitor MK-2206. Similarly, p-AKT protein expression levels increased with the addition of $50 \mathrm{nM}$ IGF-1, and decreased with the addition of 10 or $50 \mathrm{nM} \mathrm{IGF-1} \mathrm{and} \mathrm{MK-2206} \mathrm{(Fig.} \mathrm{6B).}$

\section{Discussion}

Autophagy has been reported to serve an important role in cell death $(4,6)$. The present study aimed to analyze the inhibitory 

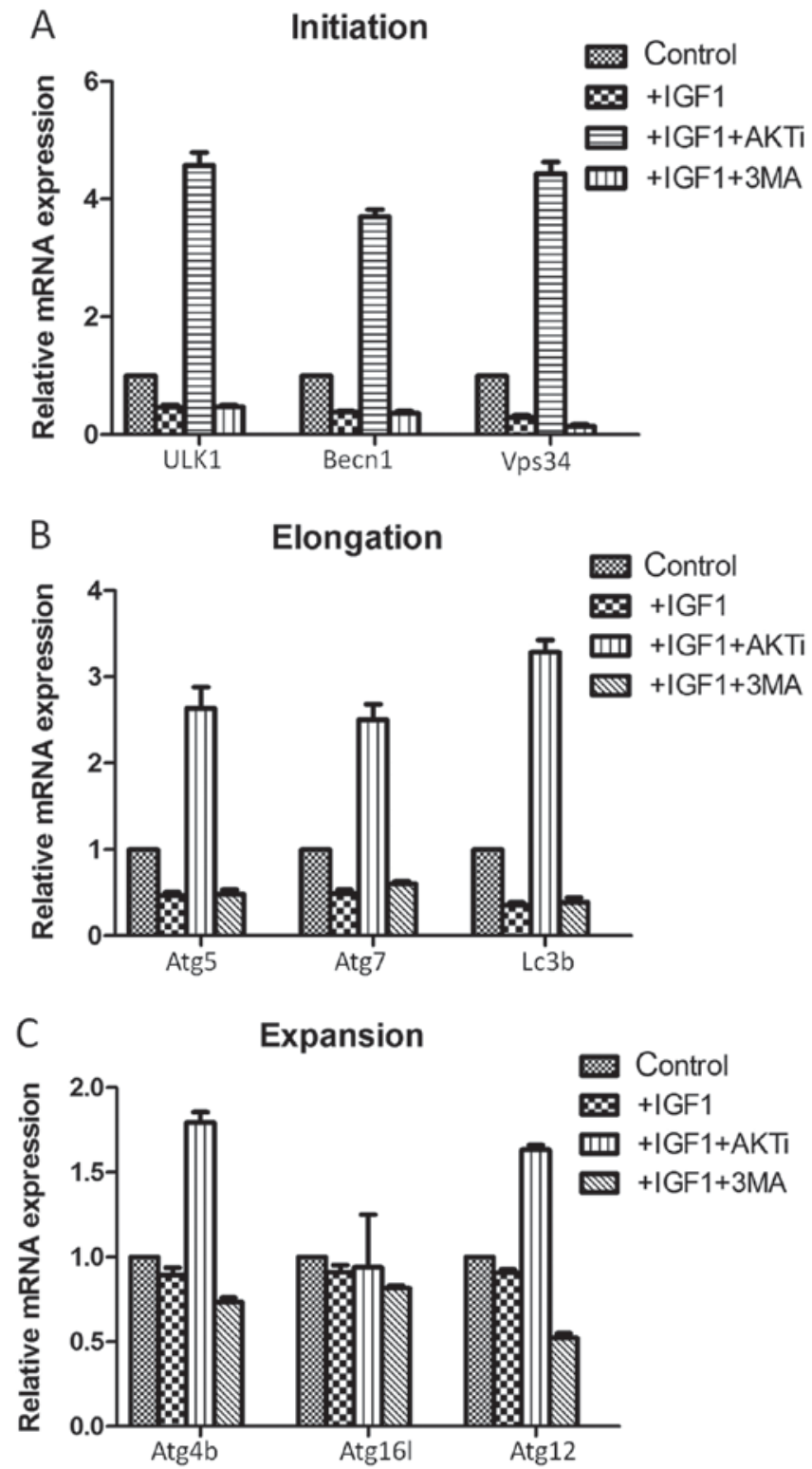

Figure 5. Reverse transcription-quantitative polymerase chain reaction analysis following treatment of cells with IGF-1, AKTi and 3-MA.mRNA expression levels of genes associated with the three stages of autophagy: (A) Initiation, (B) elongation and (C) expansion, were assessed.IGF-1, insulin-like growth factor 1; AKTi, protein kinase B inhibitor; 3-MA, 3-methyladenine; ULK1, unc-51 like autophagy activating kinase 1; Becn1, beclin-1; Vps34, phosphatidylinositol 3-kinase catalytic subunit type 3; Atg, autophagy-related; Lc3b, microtubule-associated protein 1A/1B-light chain 3. ${ }^{\# \#} \mathrm{P}<0.05$ vs. control.

effect of IGF-1 on autophagy in HCT-8R drug-resistant cells, and its potential underlying mechanisms.

In the present study, autophagic bodies decreased following IGF-1 treatment; this effect was reversed following the addition of an AKT inhibitor. This suggested that IGF-1 may inhibit autophagy by activating AKT. Through apoptosis analysis, IGF-1 was observed to increase sensitivity to apoptosis induced by 5-FU, which decreased upon the addition of an AKT inhibitor or 3-MA. These findings suggested that IGF-1 may inhibit autophagy via the AKT/mammalian target of rapamycin (mTOR) signaling pathway, and increase the sensitivity of resistant strains to 5-FU.
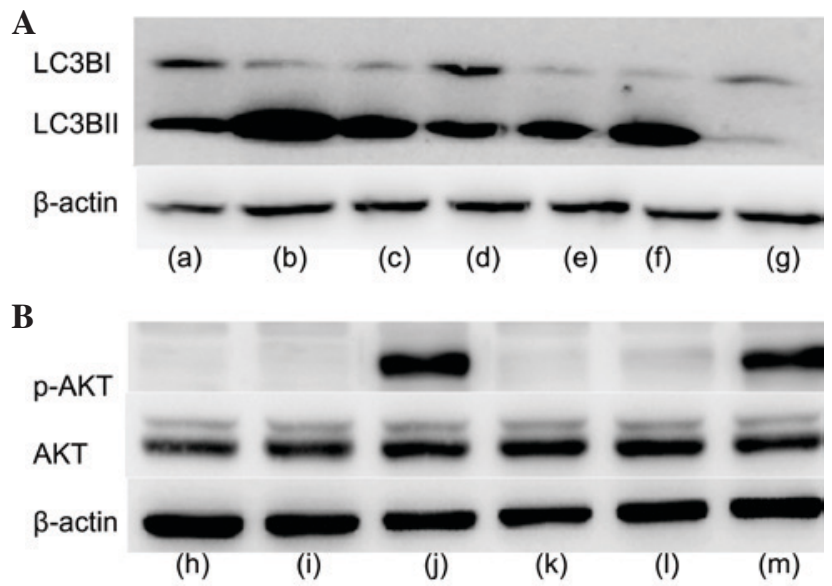

Figure 6. Western blot analysis following treatment of cells with IGF-1, AKTi and 3-MA. (A) Protein expression levels of LC3B. Lane a, FBS; lane b, FBS and AKTi; lane c, FBS and 3-MA; lane d, FBS and $50 \mathrm{nM} \mathrm{IGF-1;} \mathrm{lane} \mathrm{e,} \mathrm{FBS}$ and $50 \mathrm{nM}$ IGF-1 and AKTi; lane f, FBS, 50 nM IGF-1 and 3-MA; lane g, CM. (B) Protein expression levels of p-AKT and AKT. Lane h, FBS; lane i, FBS and AKTi; lane j, FBS and 50 nM IGF-1; lane k, FBS, 10 nM IGF-1 and AKTi; lane 1, FBS, 50 nM IGF-1 and AKTi; lane m,CM.IGF-1, insulin-like growth factor 1;AKTi, protein kinase B inhibitor; 3-MA, 3-methyladenine; LC3B, microtubule-associated protein 1A/1B-light chain 3; FBS, fetal bovine serum; CM, complete medium; AKT, protein kinase B; p, phosphorylated.

In a previous study, Lyu et al (16) revealed that peiminine resulted in cell death and promoted autophagic flux in HCT-116 cells, and suggested that peiminine enhanced autophagic flux by suppressing the phosphorylation of mTOR via the inhibition of upstream signals. In addition, Troncoso et al (11) reported that IGF-1 inhibits autophagy via the 5' adenosine monophosphate-activated protein kinase/mTOR signaling pathway in addition to the AKT/mTOR signaling pathway. It has additionally been reported that inhibition of the mTOR signaling pathway induces autophagy and decreases cell viability $(17,18)$.

The present study revealed that IGF-1 downregulated the mRNA expression levels of autophagy-associated genes involved in the three autophagy stages. Atg and Becn1 are important markers of autophagy (19-24). Previously, Jia et al (25) investigated the effect of IGF-1 on the expression of the autophagy-associated genes LC3 and Becn1 in vascular smooth muscle cells (VSMCs). Transmission electron microscopy revealed significantly reduced numbers of vacuolated cells in IGF-1-treated VSMCs compared with the untreated control group, andIGF-1 was demonstrated to inhibit the expression of autophagy-associated genes via the AKT signaling pathway. In the present study, LC3B-I and p-AKT protein expression levels increased following IGF-1 treatment, and decreased upon the addition of IGF-1 and an AKT inhibitor. These findings indicated that IGF-1 did not appear to promote apoptosis alone, although it increased sensitivity to apoptosis induced by 5-FU.

In conclusion, the results of the present study suggested that IGF-1 activated AKT and inhibited autophagy via the AKT/mTOR signaling pathway. Following inhibition of autophagy, drug-resistant HCT-8R cells became sensitive to 5-FU treatment, and treatment with 5-FU in combination with IGF-1 increased apoptosis. 


\section{References}

1. Oliveira CS, Pereira H, Alves S, Castro L, Baltazar F, Chaves SR, Preto A and Côrte-Real M: Cathepsin D protects colorectal cancer cells from acetate-induced apoptosis through autophagy-independent degradation of damaged mitochondria. Cell Death Dis 6: e1788, 2015.

2. Cunningham D, Atkin W, Lenz HJ, Lynch HT, Minsky B, Nordlinger B and Starling N: Colorectal cancer. Lancet 375: $1030-1047,2010$

3. Liu W, Zhang Z, Zhang Y, Chen X, Guo S, Lei Y, Xu Y, Ji C, Bi Z and Wang K: HMGB1-mediated autophagy modulates sensitivity of colorectal cancer cells to oxaliplatin via MEK/ERK signaling pathway. Cancer Biol Ther 16: 511-517, 2015.

4. Hayashi-Nishino M, Fujita N, Noda T, Yamaguchi A, Yoshimori T and Yamamoto A: A subdomain of the endoplasmic reticulum forms a cradle for autophagosome formation. Nat Cell Biol 11: 1433-1437, 2009.

5. Mizushima N: Autophagy: process and function. Genes Dev 21: 2861-2873, 2007

6. Galluzzi L, Morselli E, Vicencio JM, Kepp O, Joza N, Tajeddine N and Kroemer G: Life, death and burial: Multifaceted impact of autophagy. Biochem Soc Trans 36: 786-790, 2008.

7. Rubinsztein DC, Codogno P and Levine B: Autophagy modulation as a potential therapeutic target for diverse diseases. Nat Rev Drug Discov 11: 709-730, 2012.

8. Zhou WH, Tang F, Xu J, Wu X, Yang SB, Feng ZY, Ding YG, Wan XB, Guan Z, Li HG, et al: Low expression of Beclin 1 associated with high Bcl-xL, predicts a malignant phenotype and poor prognosis of gastric cancer. Autophagy 8: 389-400, 2012.

9. Yang M, Zhao H, Guo L, Zhang Q, Zhao L, Bai S, Zhang M, Xu S, Wang F, Wang X and Zhao B: Autophagy-based survival prognosis in human colorectal carcinoma. Oncotarget 6: 7084-7103, 2015.

10. Guo GF, Jiang WQ, Zhang B, Cai YC, Xu RH, Chen XX, Wang F and Xia LP: Autophagy-related proteins Beclin-1 and LC3 predict cetuximab efficacy in advanced colorectal cancer. World J Gastroenterol 17: 4779-4786, 2011

11. Troncoso R, Vicencio JM, Parra V, Nemchenko A, Kawashima Y, Del Campo A, Toro B, Battiprolu PK, Aranguiz P, Chiong M, et al: Energy-preserving effects of IGF-1 antagonize starvationinduced cardiac autophagy. Cardiovasc Res 93: 320-329, 2012.

12. Ikeda H, Shiojima I, Ozasa Y, Yoshida M, Holzenberger M, Kahn CR, Walsh K, Igarashi T, Abel ED and Komuro I: Interaction of myocardial insulin receptor and IGF receptor signaling in exercise-induced cardiac hypertrophy. J Mol Cell Cardiol 47:664-675, 2009.

13. Sekharam M, Nasir A, Kaiser HE and Coppola D: Insulin-like growth factor 1 receptor activates c-SRC and modifies transformation and motility of colon cancer in vitro. Anticancer Res 23: $1517-1524,2003$
14. Yao W, Dai W, Jiang L, Lay EY, Zhong Z, Ritchie RO, Li X, $\mathrm{Ke} \mathrm{H}$ and Lane NE: Sclerostin-antibody treatment of glucocorticoid-induced osteoporosis maintained bone mass and strength. Osteoporos Int 27: 283-294, 2016.

15. Schmittgen TD and Livak KJ: Analyzing real-time PCR data by the comparative $\mathrm{C}(\mathrm{T})$ method. Nature Protoc 3: 1101-1108, 2008.

16. Lyu Q, Tou F, Su H, Wu X, Chen X and Zheng Z: The natural product peiminine represses colorectal carcinoma tumor growth by inducing autophagic cell death. Biochem Biophys Res Commun 462: 38-45, 2015.

17. Lin CW, Jan MS and Kuo JH: Exploring MicroRNA Expression Profiles Related to the mTOR Signaling Pathway in Mouse Embryonic Fibroblast Cells Treated with Polyethylenimine. Mol Pharm 12: 2858-2868, 2015.

18. Sobolewska A, Gajewska M, Zarzynska J, Gajkowska B and Motyl T: IGF-I, EGF, and sex steroids regulate autophagy in bovine mammary epithelial cells via the mTOR pathway. Eur J Cell Biol 88: 117-130, 2009.

19. Schwartz-Roberts JL, Cook KL, Chen C, Shajahan-Haq AN, Axelrod M, Warri A, Riggins RB, Jin L, Haddad BR, Kallakury BV, Baumann WT and Clarke R: Interferon regulatory factor-1 signaling regulates the switch between autophagy and apoptosis to determine breast cancer cell fate. Cancer Res 75: 1046-1055, 2015.

20. Klionsky DJ, Cregg JM, Dunn WA Jr, Emr SD, Sakai Y, Sandoval IV, Sibirny A, Subramani S, Thumm M, Veenhuis M and Ohsumi Y: A unified nomenclature for yeast autophagyrelated genes. Dev Cell 5: 539-545, 2013.

21. Liang XH, Jackson S, Seaman M, Brown K, Kempkes B, Hibshoosh $\mathrm{H}$ and Levine B: Induction of autophagy and inhibition of tumorigenesis by beclin 1. Nature 402: 672-676, 1999.

22. Betin VM, Lane JD. Caspase cleavage of Atg4D stimulates GABARAP-L1 processing and triggers mitochondrial targeting and apoptosis. J Cell Sci 122: 2554-2566, 2009.

23. Yousefi S, Perozzo R, Schmid I, Ziemiecki A, Schaffner T, Scapozza L, Brunner T and Simon HU: Calpain-mediated cleavage of Atg5 switches autophagy to apoptosis. Nat Cell Biol 8: 1124-1132, 2006.

24. Nikoletopoulou V, Markaki M, Palikaras K and Tavernarakis N: Crosstalk between apoptosis, necrosis and autophagy. Biochim Biophys Acta 1833: 3448-3459, 2013.

25. Jia G, Cheng G, Gangahar DM and Agrawal DK: Insulin-like growth factor-1 and TNF-alpha regulate autophagy through c-jun N-terminal kinase and Akt pathways in human atherosclerotic vascular smooth cells. Immunol Cell Biol 84: 448-454, 2006.

This work is licensed under a Creative Commons Attribution-NonCommercial-NoDerivatives 4.0 International (CC BY-NC-ND 4.0) License. 